Psychology of Language and Communication 2016, Vol. 20, No. 2

商

DE GRUYTER

OPEN

DOI: $10.1515 /$ plc-2016-0009

\author{
SARAH BRO TRASMUNDI, SUNE VORK STEFFENSEN \\ University of Southern Denmark, Odense
}

\title{
MEANING EMERGENCE IN THE ECOLOGY OF DIALOGICAL SYSTEMS
}

\begin{abstract}
This article is an empirically based theoretical contribution to the investigation of meaningmaking in the ecology of human interaction and interactivity. It presents an ecological perspective on meaning-making that pivots on how agents pick up information directly in their organism-environment-system; i.e. as an activity that does not presuppose inner cognitive operations. We pursue this line of thought by presenting an analysis of how a doctor and a nurse make a decision about a specific medical procedure (catheterisation) based on meaning-making activity. As we do not see meaning as a linguistic (symbolic) or a cognitive (representational) phenomenon external to an agent/user, but as emergent in coordinated interaction, we zoom in on how the practitioners recalibrate the organism-environmentsystem by shifting between a multi-agentive mode and an individual mode. We use Cognitive Event Analysis to investigate how the agents oscillate between being a multi-agent-system with shared, tightly coordinated agency and a loosely coupled dialogical system where the individuals bring forth an understanding based on their professional backgrounds and expertise. On this view, an ecological approach to meaning-making takes a starting point in how local interaction is constrained by previous events, emergent affordances in the environment, and real-time inter-bodily dynamics. Accordingly, meaning-making is seen as a joint activity emerging from the system's coordinative actions rather than as a result of individual interpretation of symbolic content.
\end{abstract}

Key words: meaning-making, Cognitive Event Analysis, ecological psychology, dialogical systems, decision-making, interactivity, distributed cognition, human coordination

\section{Introduction}

In recent years, classic theories of language as an inventory of form-meaning pairs have been disputed (Cowley, 2011; Kravchenko, 2007; Love, 2007). While

Address for correspondence: Sarah Bro Trasmundi, Centre for Human Interactivity, Department of Language and Communication, University of Southern Denmark, Campusvej 55, 5230 Odense M, Denmark. E-mail: sarbro@sdu.dk 
the rejection of classic code-based views may entail a plethora of alternative views, recent theorising tends to converge on the ecological view that language is a tool for behavioural and social coordination (Fusaroli \& Tylén, 2012; Rączaszek-Leonardi \& Cowley, 2012; Tylén, Weed, Wallentin, Roepstorff, \& Frith, 2010). The challenge for such views, evidently, is that the rejection of form-meaning pairs is asymmetric: what one discards is the fixed relation between form and meaning, but as behavioural and social coordination is deduced from observable behaviour, form (e.g. in the shape of vocal activity) survives the post-(post)structural purgatory and resurrects in recent ecological and distributed frameworks. Where does this asymmetry leave the concept of "meaning"?

In Ecological Psychology (Gibson, 1979), "meaning" is seen as direct, i.e. as unmediated by inner cognitive processes in the animal: the animal picks up relevant information in the environment, not as stimuli for perception, but as possibilities for actions. Being relative to a particular animal's needs and abilities for interacting with the environment, Gibson (1979:127) termed these possibilities affordances: "The affordances of the environment are what it offers the animal, what it provides or furnishes, either for good or ill."

If we accept the Gibsonian view, meaning is constituted by the relation between the animal and the environment. But this view is not fully in line with recent ecological approaches to language, as they trace meaning to social coordination between human beings (as the relevant animal in question). Narrowing in on human beings as the relevant locus of inquiry, meaning is at the same time traced to human-environment relations and to human-human coordination. How do these two viewpoints align? A partial answer is found in recent developments in ecological approaches to social psychology (Marsh, Johnston, Richardson, \& Schmidt, 2009; Marsh, Richardson, \& Schmidt, 2009), but as observed by Linell $(2013,2015)$ these approaches leave the agents in a theoretical interstice: They are both seen as being parts of each other's environment and as co-constituting an agentive whole (e.g., a distributed cognitive system (Hollan, Hutchins, \& Kirsh, 2000)) The first of these two poles represents a view where the two human agents engage in wilful action: encountering the other gives the agent the possibility to greet, talk, smile, etc., and as such the agents are affordances for each other's behaviour. On the second pole, the individuality of the agents is transcended as their bodies calibrate to the presence of the other: their postural sway changes (Fowler, Richardson, Marsh, \& Shockley, 2008), their heart rate changes (Konvalinka et al., 2011), and their metabolic processes change (e.g. if they lubricate or salivate). In general, the presence of the other is known to alter a person's perception (Richardson, Marsh, \& Baron, 2007), so that the dyad's joint affordances are irreducible to the sum of their individual affordances.

These considerations imply that the dynamics of a human "organism-environment-system" (Järvilehto, 1998, 2009), are not logically bipolar but tripolar. The terms "logical bipolarity" and "logical tripolarity" refer to the logical positions 
an entity can have in an organism-environment-system: on a bipolar view, it can either be (part of) the organism or (part of) the environment. On a tripolar view, the organism and the environment are supplemented by a third pole, that of a multi-agent system that is irreducible to the agent and the environment. The agent-environment dyad thus becomes an agent-system-environment triad. On this view, human beings can be part of each other's environment, they can co-constitute a multi-agent system within an environment, or they can oscillate between these two positions (cf. Figure 1). We favour the third position because human agents are living systems, and living systems are dynamic (Van Orden, Holden, \& Turvey, 2003; Van Orden, Kloos, \& Wallot, 2011). If the agents oscillate between these two positions, the totality of the system oscillates between two states: In one state, the two agents constitute a multi-agent system that is in a state of coordination, which prompts the agents to co-orient to their joint environment. In the other state the two agents orient to each other as parts of each their environment. In the first state, their meaning-making is invested in the environment, whereas it is directed towards each other in the second state. Given that organism-environment systems are multiscalar, these two logical positions can co-exist: $\mathrm{A}_{1}$ and $\mathrm{A}_{2}$ can synchronously coordinate and co-orient; they can fluctuate between coordinating and being coordinated. ${ }^{1}$

Figure 1. Oscillations in a human organism-environment system. Seeing one agent $\left(A_{1}\right)$ as a fixed point allows for seeing the other $\left(\mathrm{A}_{2}\right)$ as oscillating (along the arrow) between co-constituting a multi-agent system (at the left pole) and being part of $\mathrm{A}_{1}$ 's environment (at the right pole). The inter-agentive dynamics are here described as a movement on the part of $\mathrm{A}_{2}$ : the "centripetal" movement gives rise to a multi-agent system, whereas the "centrifugal" makes the two agents orient to each other as parts of their environment

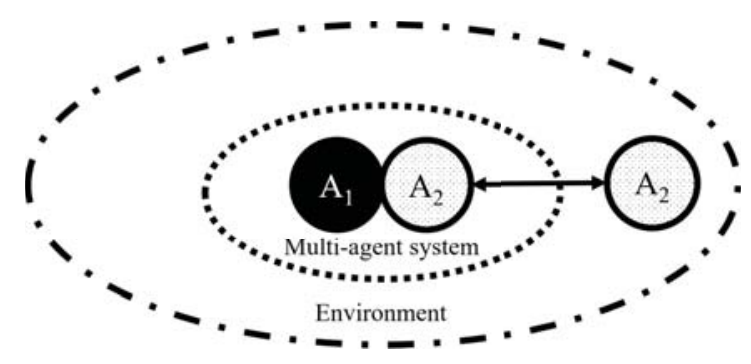

\footnotetext{
${ }^{1}$ This understanding of multi-agent systems resembles the theoretical models of "participatory sense-making" (Cuffari, Di Paolo, \& De Jaegher, 2015; De Jaegher \& Di Paolo, 2007), "distributed cognitive systems" (Hollan et al., 2000), "transient extended cognitive systems" (Wilson \& Clark, 2009), and "dialogical systems" (Steffensen, 2012). Space limitations prevent us from an in-depth discussion of the similarities and differences, but we see the present article as a contribution to the dialogical systems thinking.
} 
These considerations have made us hypothesise that the kind of "meaning" that led the tradition to assume a semantic realm, can be traced to these oscillations in tripolar dialogical systems: in the systemic mode, the system seeks to achieve results in a shared environment, which requires that they invest their coordination-induced meaning in environmental affordances. In contrast, the "centrifugal" movement entails a state where the agents orient to each other as dialogical affordances for meaning. In either state, the affordances depend both on the dialogical system's history of coordination, and on the agents' autobiographical backgrounds.

This view on meaning corresponds to the intuition that led Karl Bühler (1934) to suggest his organon model. To Bühler, signs both connect the interlocutors (our "multi-agent system") and point to an external world ("the environment"). But whereas Bühler couched his model in terms of representational signs that function as communicative mediators between a sender and a receiver, we emphasise that "meaning" is not a quality of independently given signs. Rather meaning emerges in the dynamic interplay between agents, and between these agents as a dialogical system and their joint environment. In ecological psychology, the epistemological interest has been invested in the understanding of meaning in agent-environment relations, whereas social psychology has invested their epistemological interest in agent-agent dynamics. In line with recent developments in ecological linguistics, we insist that we need to bring these two dimensions of meaning together.

Interestingly, the two dimensions correspond to the two semantic traditions mentioned by Sidnell (2016), namely "the designative tradition" (meaning related to the environment) and "the expressive tradition" (meaning related to the socially embedded communicators). But our model transcends both of these traditions, as we insist that meaning is not a quality of signs, but deeply interwoven with the ways in which agents are embedded in an environment - that is, with their interactivity with a term we have proposed elsewhere (Harvey, Gahrn-Andersen, \& Steffensen, 2016; Pedersen, 2012, 2015; Steffensen, 2013, 2015; Steffensen, Vallée-Tourangeau, \& Vallée-Tourangeau, 2016). As such, we see our ecological research agenda as aligned with Sidnell's when he remarks that "both these approaches, and others as well, never ask what meaning amounts to as a practical matter of daily life and ordinary talk. That is, what does 'meaning' do?" (Sidnell, 2016). Likewise, we align with Sidnell's position when he argues that such questions can only be answered by taking an empirical approach to meaning-making, i.e. by investigating how human beings understand a given situation through their coordinative and coordinated actions.

In this article we pursue the question of what meaning does in a practical setting, namely in a department of emergency medicine (Pedersen, 2015). However, our approach differs from Sidnell's in two ways. First, whereas Sidnell (2016) asks "How are word meanings actually used by persons in the course of speaking to 
one another?," we do not see meanings as pertaining to words or other constructs from the linguistic tradition, and we do not see meaning as something that can be used, as that implies that 'meaning' has an externalised, independent existence apart from 'the user'. Quite obviously, we do not intend to say that words etc. are "meaningless" or unrelated to meaning dynamics. Meaning emerges in dialogical systems, and it is irreducible to any single part of these: even if extending the middle finger upwards towards your interlocutor is routinely associated with an obscene symbol of disrespect, the finger is not the carrier of that meaning. The same goes for the production of sequences of recognisable sounds, such as /'minin/. It is not the sound sequence itself that means 'meaning', but within the totality of the dialogical system, the specific behaviour (including the vocalisation of /'minin/) gives rise to certain behavioural outcomes across the system. As argued by Wittgenstein half a century ago, it is a fallacy to isolate the "word" (or the gesture) as the carrier of meaning. But on the other hand, if an observer takes a "language stance" (Cowley, 2011), according to which such vocalisations are heard as symbols, s/he can see a correlation between how the dialogical system changes and how vocalisations emerge in this course. In other words, even if the observer denies that meaning pertain to words and symbols, s/he can still use such symbols as heuristic tools for understanding what happens in the dialogical system. Further, as also the participants in the dialogical system observe their own, and each other's, behaviour, they too take a language stance that allow them to exploit the energy condensed in symbols: symbols become nudges capable of co-constraining and co-controlling dialogical systems, not as structures sui generis, but as emergent behaviours within the system (cf. Rączaszek-Leonardi, 2011). Hence, whether it is traced to behaviour, symbols or an attractor on a longer timescale, meaning emerges in the ecology of dialogical systems. In our terminology, we would say that the dialogical system becomes "sense-saturated" (Steffensen, 2013). ${ }^{2}$

Second, we are less interested in "the course of speaking to one another" per se, than in the ecological implications of such interactions, i.e. in how dialogical systems achieve results in a larger ecological environment. This emphasis on the environmental implications of dialogical systems reflects our approach to 'meaning' as equally invested in agent-agent interaction and in agent-environment interactivity. On this view, meaning is irreducible to coordination between the interlocutors, because the purpose of interaction lies beyond interaction. To Rączaszek-Leonardi and colleagues (2014), the purpose of interaction is twofold: "to maintain the systemic character of a conversing dyad and to organize it into a functional synergy in the face of tasks posed for a dyadic system as a whole." In general, linguists and interaction analysts have been reluctant to dive into the task component in dialogical systems, in part for methodological reasons, as

\footnotetext{
${ }^{2}$ On an evolutionary timescale, when the dynamics of a dialogical system became saturated by meaning, the action potentials within such systems were radically transformed (Rączaszek-Leonardi, 2011).
} 
this component requires a cognitive/psychological theoretical frame that allows one to investigate how human agents engage in the environment. Two areas of cognitive psychology come to the fore as particularly relevant in this context: problem-solving psychology and decision-making psychology, as they both describe how subjects approach external tasks and problems. The scope of this article prevents us from an in-depth incorporation of any of these fields; in what follows we scrutinize an example where the emergence of meaning plays out in a process that can be aptly described as joint decision-making. The crucial observation made here is that our approach to 'meaning' involves the tripolar dynamics of two agents and an environment, which also goes for joint decision-making. 'Decision-making' is a cognitive process during which the agent(s) identifies various alternatives present in the environment and weighs these alternatives based on goals, values and preferences, and joint decision-making is thus the same process where the decision process is driven by a multi-agent system. While the decision-making process can be investigated from a psychological point of view, only an approach that acknowledges meaning-making processes can grasp the dynamics within the dialogical system as well as the results achieved. When a dialogical system faces a situated need to make a decision, its only way forward involves the kind of dynamics that, from the ecological point of view sketched above, will give rise to meaning.

To pursue this line of research, we will first (in section 2) present the method applied in this article, namely that of Cognitive Event Analysis (Steffensen, 2013, 2016; Pedersen \& Steffensen, 2014; Steffensen et al., 2016; Vallée-Tourangeau, Steffensen, Vallée-Tourangeau, \& Makri, 2015). Second, in section 3 we apply our ecological framework to a single case study from a Danish emergency medical department. After a short intermezzo (in section 4), where we relate our case to the participants' experience as expressed in a post hoc interview, we conclude (in section 5) by discussing our results vis-à-vis the overall question of this special issue: what is "meaning" in an ecological view on language?

\section{Cognitive Event Analysis}

Cognitive Event Analysis is an ecological method for investigating how humans adapt to and modify their environment in order to move forward and get things done (Steffensen, 2013). Space limitations prohibit a thorough unfolding of the analytical method (for a detailed presentation, see Cowley \& Nash, 2013; Pedersen, 2015; Steffensen, 2013, 2016; Steffensen et al., 2016; Trasmundi \& Linell, submitted). CEA pivots on a fine-grained examination of video recordings of human interactivity. Building on a qualitative and cognitive basis, it investigates how human organism-environment systems achieve results. More specifically, it scrutinises the changes and transitions in the interactivity trajectory that emerge around cognitive activities. With a focus on how results are achieved 
(e.g. how problems are solved or how decisions are made), it investigates the relevant processes of embodied meaning making that lead to particular actions and outcomes in the system. The methodological procedure of CEA consists of multiple steps spanning from event identification to interpretation (see Steffensen et al, 2016). An important part of this procedure is the identification of phase transitions including event pivots. Phase transitions indicate changes in the flow of interactivity, e.g. when the system oscillates between coordinated and less coordinated states. Such changes in the oscillation pattern are indicative of the agents' movements within the system, and they are bound up with how meaning contributes to the recalibration of the system. Event pivots are crucial transition points where changes in the dynamics of the dialogical system has a cognitive function vis-à-vis the task undertaken by the system. Examples include how a team experiences a breakthrough while working on a challenging problem, or how it suddenly comes to see something odd as fitting the larger picture, or when it reaches a decision. CEA focuses on connecting such event pivots to explain how results are achieved in dialogical systems. In the current context, we zoom in on inter-bodily coordination pertaining to how meaning-making emerges in situ.

CEA prompts the analyst to deal with multiple timescales. While the method pays particular attention to real-time bodily dynamics playing out in local coordination, it links those behavioural patterns to longer timescales beyond real-time interaction, for instance when sociocultural timescales come to the fore as an individual's skilful behaviour.

\section{Meaning dynamics in a dialogical system: the medical team in action}

Having presented CEA, we now turn to a case study where we focus on the tripolar dynamics that constitutes meaning (cf. section 1). In our study, we are specifically interested in how the two agents in our example shift from a state of being a coordinated medical team to a state of negotiating what to do next.

The example comes from ethnographic work done by Trasmundi (Pedersen, 2015) in an emergency medical department at a hospital in the Copenhagen area in Denmark. In this article, we focus on a situation where a 92-years-old woman has been hospitalised with a proximal femur fracture (a hip fracture). At the hospital she is received by a medical team that consists of an experienced nurse and a novice doctor, who had worked at the ward for only a couple of days when the recordings took place. The asymmetry between the two practitioners leaves them in a precarious position: on the one hand, the role of the "doctor" entails certain legal and cultural obligations to take the lead, ${ }^{3}$ and on the other hand,

\footnotetext{
${ }^{3}$ In a Danish context, these cultural expectations are sometimes referred to as doctors having a "Tarzan syndrome": they are expected to deal with everything themselves, and asking for help is a sign of weakness.
} 
newcomers in teams tend to take a position of "legitimate peripheral participation" (Lave \& Wenger, 1991). One would expect that this tension between two sets of expectations on a cultural timescale plays out in the interpersonal dynamics on an interactional timescale (Steffensen \& Pedersen, 2014). An overview of the setting is given in Figure 2.

Figure 2. Overview of the layout. In the "centripetal" state the two health practitioners are oriented to the patient as an affordance for taking medical action, and in the "centrifugal" state the two are oriented to each other in a meaning-inducing intermezzo in the medical process. In this figure, the simultaneity of the two states is visible in the way that hand gestures are oriented towards the patient, while gaze is oriented to each other

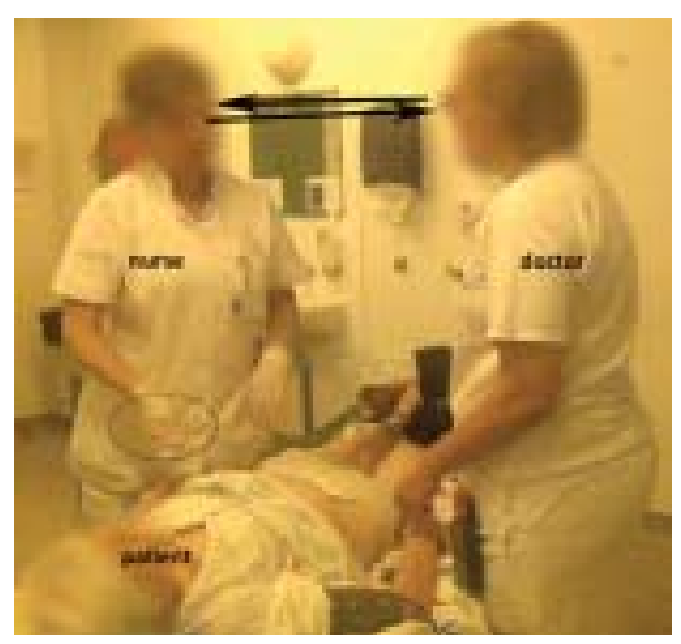

As the patient has been diagnosed with a hip fracture, the treatment of the patient will include orthopaedic surgery. However, before the patient is ready for that, the medical team has to go through a number of pre-treatment procedures, including pain alleviation and stabilisation of the patient's other bodily functions. Our focus is on a single procedure in the pre-treatment phase that relate to the latter, namely the patient's ability to urinate. In our case, the medical team - in accordance with standard procedures - performs a urinary catheterisation. The urinary catheterisation is a rather trivial procedure, but the decision to perform it depends, not on rules, but on a judgment based on the mobility of the patient. In our case, this decision becomes an interesting cognitive event 
because the team, to begin with, decides not to perform the procedure in spite of a high number of indications that catheterisation is indeed necessary. The event illustrates how meaning in the dialogical system emerges in the interplay between sociocultural regularities, individual expectations, knowledge about medical procedures, perceptual pick-up of information about the patient, and situated decision-making.

From a procedural point of view, a decision to perform a urinary catheterisation has three steps which are all saturated with meaning-making: first, the team must identify a need (i.e., it must come to see it as a meaningful procedure to consider); second, they must examine a number of informed arguments pro and con the procedure; and third, the team, or one of the participants in it, must conclude (either through wordings or through action) that the arguments in favour of catheterisation outweighs those against. In the vast majority of our ethnographic material, these steps are collapsed into a single action: practitioners "just know" that catheterisation will be needed sooner or later, and the arguments in favour of the procedure are so well-established that it is hardly even seen as an argumentation. Accordingly, the procedure is "just done." However, in this case the medical team diverts from the three procedural steps; they go through the second step two times: the first time (which is the focus of this article) they do not reach the conclusion that they need to insert a catheter, whereas they do so the second time (at a later stage in the pre-treatment procedure). As the decision not to perform a urinary catheterisation significantly diverts from the standard medical procedures at the department, the question from a cognitive point of view obviously becomes: what happened when they decided not to catheterise the patient?

In what follows we will analyse this event in fine-grained detail, but let us first have a look at the app. 60 seconds in which the relevance of catheterisation and the decision not to catheterise play out. For a more accessible overview, we first rely on a transcription of the episode: ${ }^{4}$

Transcript: Duration 01:01:00 minutes

\section{DANISH ORIGINAL}

$\begin{array}{lll}\text { 1. } & 34: 01: 10, \mathrm{P}: & \text { jeg har s jeg har sådan en (.) ble p [å } \\ \text { 2. } & 34: 03: 50, \mathrm{D}: & \\ \text { 3. } & 34: 04: 60, \mathrm{P}: & \text { ja } \\ \text { 4. } & 34: 05: 00, \mathrm{ps} & (0.8) \\ \text { 5. } & 34: 05: 80, \mathrm{D}: & ø \mathrm{hm} \\ \text { 6. } & 34: 06: 40, \mathrm{ps} & (0.7) \\ \text { 7. } & 34: 07: 10, \mathrm{~N}: & \text { vi skulle jo nok lægge et kateter } \\ \text { 8. } & 34: 08: 60, \mathrm{ps} & (0.9) \\ \text { 9. } & 34: 09: 50, \mathrm{D}: & \text { ska vi det? (.) } \\ \text { 10. } & 34: 10: 20, \mathrm{~N}: & \text { eller (.) det er ikke nødvendigt måske }\end{array}$

\footnotetext{
${ }^{4}$ We apply the transcription system developed by Gail Jefferson for the analysis of conversation (Jefferson, 2004). $\mathrm{D}$ is the doctor; $\mathrm{N}$ is the nurse; $\mathrm{P}$ is the patient.
} 
11. $34: 12: 30, \mathrm{P}$ :

12. $34: 12: 60, \mathrm{D}:$

13. $34: 15: 30, \mathrm{~N}$ :

14. 34:19:20, ps.

15. $34: 19: 90, \mathrm{~N}$ :

16. $34: 22: 80, \mathrm{P}$ :

17. $34: 23: 10, \mathrm{~N}$ :

18. $34: 26: 40$, ps:

19. $34: 27: 20, \mathrm{P}$ :

20. $34: 30: 30, \mathrm{~N}$ :

21. $34: 30: 70, \mathrm{D}:$

22. $34: 31: 80, \mathrm{~N}$ :

23. $34: 32: 40, \mathrm{D}:$

24. $34: 33: 80, \mathrm{~N}$ :

25. $34: 36: 60, \mathrm{D}:$

26. $34: 37: 40, \mathrm{~N}$ :

27. $34: 38: 40, \mathrm{~N}$ :

28. $34: 40: 20, \mathrm{P}:$

29. $34: 45: 20, \mathrm{D}:$

30. $34: 45: 80, \mathrm{P}:$

31. $34: 48: 20, \mathrm{~N}$ :

32. $34: 50: 80, \mathrm{D}:$

33. $34: 51: 30, \mathrm{~N}$ :

34. $34: 57: 90, \mathrm{D}:$

35. 35:00:10, P:

36. 35:00:70, D:

37. $35: 01: 30, \mathrm{~N}$ :

38. 35:01:70, D: $(\mathrm{xxx})$ [det det det næ det plejer jeg [ikke [na:rj det er da ikke nødvendigt [hvis ikke du plejer at bruge kateter

[nej jeg tænker nogen

gange med mobilisering til bækken og sådan noget men det kan vi jo nok godt $\downarrow$

(0.7)

få et bækken ind under når du skal tisse og sådan $\uparrow$

ja [det ved jeg-

[men det virker som du er rimelig god alligevel til og lige at få $(0.8)$

ja bortset fra at $ø$ den der den gør ondt

ja:=

=men nu lægger jeg jo FIC-blokken

ja

tror du tror du ikke vi klarer $\left[{ }^{\circ o} u d e n^{\circ o}\right.$

[vi gør det jo tit men men men lad os bare prøve at se

ja det ved jeg ik så [det det er jo det kan da godt være vi [ska det er dig øh

[ja men

det er jo folk der [ik øh ka:: ka bevæge sig så godt og jeg synes egentlig hun er rigtig god

med min mave

[altså jeg har jeg har jeg har den der på gulvet

med maven [der driller den er [lidt tynd (.) ja

[ja [ja ja

lad os bare lige øh (.) nu prøver vi bare li:ge at lægge det her så

e ellers det [jeg tror det er dig de::r er me::st øh

[ja jeg tror nemlig (0.1) ja (0.1) vi ser det lige an (0.3)

fordi jeg synes egentlig også at øh det er realistisk og

det er jo begrænset hvor mange bækkener jeg lægger

ja

eller hvad hedder det

ja (smiling)

så (laughs)

\section{ENGLISH TRANSLATION}

1. 34:01:10, P:

2. $34: 03: 50, \mathrm{D}:$

3. $34: 04: 60, \mathrm{P}$ :

4. 34:05:00, ps.

5. $34: 05: 80, \mathrm{D}$ :

6. $34: 06: 40$, ps.
I have a I have such a (.) diaper o [n

[ye::s you have

yes

(0.8)

ehm

(0.7) 
7. $34: 07: 10, \mathrm{~N}$ :

8. $34: 08: 60$, ps.

9. $34: 09: 50, \mathrm{D}:$

10. $34: 10: 20, \mathrm{~N}$ :

11. $34: 12: 30, \mathrm{P}:$

12. $34: 12: 60, \mathrm{D}:$

13. $34: 15: 30, \mathrm{~N}$ :

14. $34: 19: 20$, ps.

15. $34: 19: 90, \mathrm{~N}$ :

16. $34: 22: 80, \mathrm{P}$ :

17. $34: 23: 10, \mathrm{~N}$ :

18. $34: 26: 40$, ps.

19. $34: 27: 20, \mathrm{P}$ :

20. $34: 30: 30, \mathrm{~N}$ :

21. $34: 30: 70, \mathrm{D}:$

22. $34: 31: 80, \mathrm{~N}$ :

23. $34: 32: 40, \mathrm{D}:$

24. $34: 33: 80, \mathrm{~N}$ :

25. $34: 36: 60, \mathrm{D}:$

26. $34: 37: 40, \mathrm{~N}$ :

27. $34: 38: 40, \mathrm{~N}$ :

28. $34: 40: 20, \mathrm{P}:$

29. $34: 45: 20, \mathrm{D}:$

30. $34: 45: 80, \mathrm{P}$ :

31. $34: 48: 20, \mathrm{~N}$ :

32. $34: 50: 80, \mathrm{D}:$

33. $34: 51: 30, \mathrm{~N}:$

34. 34:57:90, D:

35. 35:00:10, P:

36. $35: 00: 70, \mathrm{D}:$

37. $35: 01: 30, \mathrm{~N}$ :

38. 35:01:70, D: we should probably insert a catheter

(0.9)

we should? (.)

or (.) that is not necessary perhaps

$(\mathrm{xxx})$ [that that that no: I do

[no:: that should not be necessary

usually use a catheter

[not

[if you do not

[no I think

sometimes with regards to the mobilisation to the bedpan and such but I guess we can manage that $\downarrow$

(0.7)

place a bedpan under when you need to go to the toilet and such $\uparrow$ yes [I don't know

[but it seems like you are pretty good anyway to just get

yes except from ehm that this one it hurts

ye:s=

=but now I am going to perform the FIC block right

yes

don't you don't you think we manage $\left[{ }^{\circ o}\right.$ without ${ }^{\circ o}$

just try and see

[we often do it but let us

yes that I do not know so [it it is well it might be that we [should that is you eh

[yes

[yes

but that is people who are [unable eh to move so easily and I actually think she is rather mobile

[well I have I have I have this one on

the floor

with the stomach [that is a bit tricky it is [a bit diarrhoea like (.) yes [yes [yes yes

let us just eh (.) now we are ju:st trying to give this so

o or this [I think it is you who a::re the mo::st eh [yes because I believe (0.1) yes (0.1) we will just wait and see $(0.3)$ because I actually also think that eh it is realistic and after all I only place a limited number of bedpans

yes

or what is it called

yes (smiling)

so (laughs)

By matching the procedural description of the three steps in decision process with the transcript we identify the following key transition points in the cognitive trajectory: (i) the relevance of dealing with the patient's urinary function emerges 
in line 1 (P: I have such a (.) diaper on); (ii) the initial suggestion to catheterise is expressed by the nurse in line 7: we should probably insert a catheter, and (iii) the decision to rely on using a bedpan for her urination is taken in line 33 when the nurse says: we will just wait and see (0.3) because I actually also think that eh it is realistic. In the terminology of CEA, these three transition points are event pivots: they indicate when the problem becomes relevant, when the argumentation begins, and when a decision is reached and confirmed.

What makes this particular event so interesting is that the nurse's initial judgment will be carried out much later in the pre-treatment process, but it is overruled in this sequence, as they reach the conclusion not to catheterise and rather rely on a bedpan. As this outcome is a direct result of the meaning processes in the dialogical system, the emergence of meaning is best investigated by zooming in on the relevant event pivots and transition points that indicate changes in the dialogical system's specific meaning configuration, and hence the focus on these transition points gives insight into the overall meaning-making trajectory.

Investigating the cognitive trajectory, in particular step 2 in the decision process (i.e. the sequence in line 7-33), thus gives us the opportunity to investigate how the two agents create different meanings (understood as organism-environment relations) and how they through meaning coordination reach a decision. Thus, based on changes in the agents' meaning-making in relation to the task, we segment this sequence into seven phases (see Table 1).

Table 1. Seven phases in the meaning configuration in the dialogical system

\begin{tabular}{|c|c|c|}
\hline Phase & Line & Meaning configuration \\
\hline I & $7-12$ & $\begin{array}{l}\text { The nurse suggest catheterisation } \\
\text { The doctor does not concur with catheterisation }\end{array}$ \\
\hline II & $13-18$ & $\begin{array}{l}\text { The nurse repeats her suggestion to catheterise the patient } \\
\text { The doctor is silent }\end{array}$ \\
\hline III & $19-23$ & The catheterisation suggestion is related to the patient's pain \\
\hline IV & 24 & $\begin{array}{l}\text { The catheterisation suggestion is related to the department's } \\
\text { procedures }\end{array}$ \\
\hline $\mathrm{V}$ & $25-27$ & $\begin{array}{l}\text { The doctor reconsiders catheterisation } \\
\text { The nurse argues against catheterisation }\end{array}$ \\
\hline VI & $31-32$ & $\begin{array}{l}\text { The doctor reconsiders catheterisation } \\
\text { The nurse argues against catheterisation }\end{array}$ \\
\hline VII & 33 & The nurse concludes that catheterisation is not necessary \\
\hline
\end{tabular}


The seven phases are visualised in Figure 3 below. In what follows, the trajectory described in Table 1 and Figure 3 will be elaborated in detail by zooming in on each of the seven phases.

Figure 3: the emergence of meaning in decision-making. The arrow shows the cognitive trajectory, the blue triangles indicate event pivots, and the white triangles indicate phase transitions. The phase transition points and event pivots segment the trajectory into the seven phases listed in Table 1

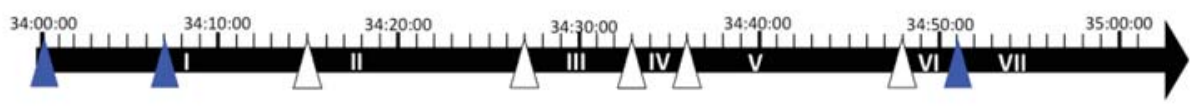

\section{Phase I: Making medical procedures meaningful}

The first phase concerns the nurse's proposal to consider urinary catheterisation. Her proposal is prompted by the patient mentioning that she is wearing a diaper (line 1). This utterance is identified as an event pivot, because it prompts the nurse to interrupt the procedural flow in the medical team by suggesting catheterisation as the proper solution to an anticipated problem of urination.

In theoretical terms, the coordinated multi-agent system is perturbed, and the two practitioners re-orient to each other as relevant affordances for their meaning-making activities. This re-orientation is observable from the nurse's gaze behaviour. Thus, when the nurse begins on her proposal (in line 7), she gazes at the patient's body, but at the end of the utterance, her gaze is redirected to the doctor. Hence, the dialogical system undergoes a centrifugal movement where they move from a coordinated state with joint attention to a less-coordinated state where they need to achieve a shared interpretation of the situation. This process of coordination depends on a centripetal movement that gives rise to meaning dynamics in the situation, and from an observer's point of view, this meaning is observable in their bodily orientation. Thus, the nurse makes the hip area of the patient's body relevant through her gaze, and both doctor and nurse pay attention to that part of their environment, which hence becomes a shared affordance for meaning and subsequent action. The nurse's combination of verbal prompting and gaze becomes an affordance for restoring the multi-agent system: the nurse makes it clear what action must be initiated, and by pointing to relevant features in the environment, she prompts the doctor to perceive the same meaning. But the doctor does not follow the nurse's lead. After a pause of 0.9 seconds (line 8), the doctor questions the suggestion explicitly: We should? 
(line 9). Thus, the patient's initial utterance becomes an affordance for very different action possibilities. In this way several meanings emerge: The nurse argues for catheterisation, the doctor does not concur. ${ }^{5}$

Since the decision on whether to catheterise or not is based on how mobile the patient is, the doctor's utterance we should? (line 9) indicates that catheterisation was not part of the doctor's pre-treatment plan, either because she does not share the nurse's judgment, or because she never even thought of it as a relevant procedure to consider. Accordingly, the doctor's hesitation creates a relational ambiguity: on the one hand, if the doctor reject's the nurse's judgment, the role hierarchy and cultural expectations dictate that the doctor has the right and obligation to make the final decision; on the other hand, if the doctor's reaction is caused by the fact that she did not take a relevant procedure into consideration, priority is normally given to the one in the best position to overview the situation, and in this case that is the most experienced participant: the nurse. Evidently, the ambiguity cannot be easily resolved, and hence two meanings co-exist in an interpersonal negotiation that needs to balance both a clinical aspect and a cultural role aspect.

From the nurse's subsequent utterance - or (.) that is not necessary perhaps (line 10) - it is clear that she picks up on the first of these two aspects: she modulates her proposal into a question, so while she initially proposed a procedure, she now asks the doctor whether this procedure is a good idea. While the doctor's contribution to the reasoning has been minimal, the sequence has prepared a conclusion (catheterisation is not necessary) and a line of argumentation (a rejection of the nurse's judgment) that the doctor is prompted to adopt. It is not based on an elaborate argumentation, however, but such an argument is quickly reconstructed. To come up with an argument, the doctor uses the patient as a cognitive resource (line 10 and 11). The patient is made relevant by the nurse: when she utters that catheterisation is perhaps dispensable (line 10), her gaze moves from the doctor to the patient. In this way, the patient becomes involved in the negotiation, and she states that she does not usually need a catheter. The doctor uses this statement and frames it as a warrant for her own opinion (line 12): no:: that should not be necessary if you do not usually use a catheter. As the doctor addresses the patient with the deictic 'you', she changes her orientation (in gaze, in posture, and in verbal behaviour) from the nurse to the patient, leaving the nurse as a less significant component in the system as she becomes a temporary observer of what is going on.

The information provided by the patient has a significant impact on the meaning processes in the interaction. It confirms the doctor's line of argumentation,

\footnotetext{
${ }^{5}$ As pointed out by Joanna Rączaszek-Leonardi (pers.comm.) this segment of our example is a powerful illustration of the importance of social systems and sociocultural norms and regularities. Thus, the very fact that the patient's initial utterance has such a big impact on the overall trajectory is related to specific Danish norms of non-hierarchical egalitarianism and inclusion.
} 
and because the multi-agent dialogical system so crucial in medical teamwork is dissolved, the role hierarchy means that the doctor is positioned as the main cogniser; i.e. as the one who is going to make the decision. However, from a medical point of view, the patient's information is utterly useless: whether the patient needs a catheter or not, is not a question of whether the patient normally uses one, but only a question of her present condition. The only relevant question is whether her broken hip complicates or even prevents her from going to the toilet or using a bedpan or not.

In conclusion, including the patient as a relevant source of information in the decision-making process does not contribute any valuable information regarding the clinical problem at hand. However, integrating the patient has a direct impact on the oscillations in the system, as it enables the doctor to dissolve the initial meaning ambiguity by reaching a conclusion in a way that is consistent with the provided, though irrelevant, information. In this way, she avoids rejecting the nurse's proposal, but the price is high: she relies on irrelevant information and her conclusion is invalid.

\section{Phase II: Embodiment as an affordance for meaning}

In the cultural context of a medical department in Denmark, the doctor is the main responsible for patient treatment, and in the end she has the authority and the obligation to decide what to do. Offhand, it seems as if a decision has already been reached in the previous phase: the nurse proposed a pre-treatment procedure, but the doctor rejected it. In our ethnographic material it is extremely rare that such decisions are questioned, and if one reads the transcript of the second phase, which runs from line 13-18, it shows that the nurse on two instances concurs with the doctor's decision: but I guess we can manage that $\downarrow$ [and] place a bedpan under when you need to go to the toilet and such $\uparrow$ (line 13-15) and it seems like you are pretty good anyway to just get (line 17). But she also expresses her disagreement with the emerged conclusion: First, for the only time during this sequence, the nurse interrupts another interlocutor, namely the patient. Not only does she interrupt the patient, she does so while the patient is sharing her experiences with using a catheter (line 11), which, as we saw, is the utterance that implicitly, and logically fallaciously, supported the doctor's hesitation. Second, the nurse expresses an outright disagreement: no (kursywą) she says in line 13 in response to the emerged conclusion in the first phase. Third, she expresses a vague concern with the implications of the conclusion: I think sometimes with regards to the mobilisation to the bedpan and such (line 13).

Her concerns are vaguely phrased, but they are accentuated and amplified by her embodied behaviour. Thus, the phase transition marks a change from verbal argumentation in Phase I to embodied performance and visualisation in Phase II. This change in the trajectory comes to the fore when she anticipates the line of actions in case the patient needs to urinate. This anticipation is articulated in 
a whole-bodied narrative of how the bedpan is difficult to place under an impaired patient. She refers to her experiences from previous situations with the words sometimes with regards to the mobilisation (line 13), and at the same time she engages in an embodied visualisation of the physical constraints in placing a bedpan under the patient. We scrutinise the nurse's visualisation in the following (cf. picture A-D in Figure 4 below).

Figure 4. Decision-making in Phase II. In picture A-D, the nurse wiggles her hips and torso from side to side as she moves her right hand back and forth. These body movements visualise the physical constraint when arranging a bedpan. Simultaneously, the nurse utters: no I think sometimes with the mobilisation to the bedpan and such (line 13)

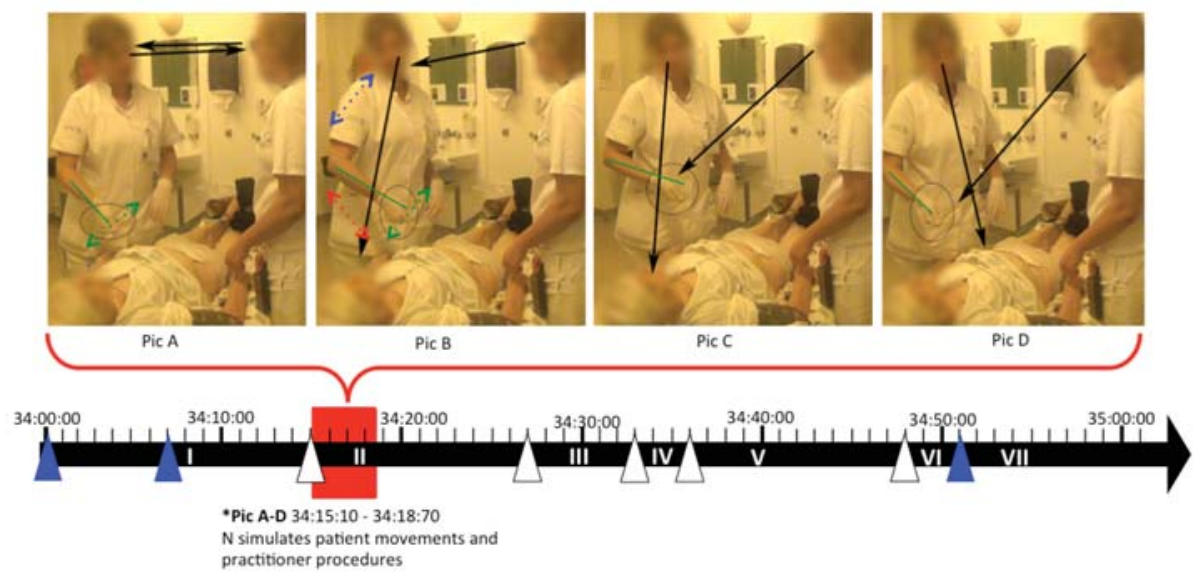

The nurse performs the situation for both the doctor, whom she needs to convince in order to make the right decision, and the patient, who will feel the consequences of the decision. As the nurse verbally refers to the process of placing the bedpan (line 13), she performs a series of embodied movements: she wiggles her hips and upper body from side to side while she moves her right arm back and forth. The arm movements simulate the actual bodily movements required in placing a bedpan under the patient (see Figure 4), and the simultaneous hip wiggling illustrates the movements of the patient during the process of placing the bedpan. In other words, in this small scenario the nurse performs the actions of two agents: the nurse placing the bedpan and the patient moving her (fractured) hip. The nurse simultaneously communicates the bodily consequences to the patient and the constrained working condition to the doctor. Using her 
own body as a display, she creates affordances for perceiving the future consequences of a particular procedure. By so doing, she prompts the dialogical system to restore a joint meaning and a joint interpretation. This centripetal movement is seen in Figure 4, where the doctor first gazes at the nurse's head (picture A-B) but soon after turns her attention to the movements that the nurse performs (picture C-D). Such a reconfiguration in the dialogical system, where the participants attend to each other's embodied behaviour, gives rise to new patterns of meaning-making: they temporarily suspend their orientation to the task and the patient in order to calibrate how they perceive the problem of urination and the potential solution of catheterisation. In the overall treatment procedure, verbal symbols have functioned as nudges that impacted on their joint orientation to the patient (Rączaszek-Leonardi, 2011), but now meaning-making emerges from the embodied behaviour as they orient to each other. During the narrative the doctor carefully observes how the nurse plays out the scenario through embodied movements. When the nurse has demonstrated the problem, she stops moving her body. Interestingly, in immediate continuation of this dramatised intermezzo, the nurse contradicts her own concern by estimating that they probably can mobilise the patient in order to place the bedpan: but I guess we can manage it $\downarrow$ (line 13) as she nods 'yes' and gazes at the doctor. So while she creates the bodily affordances for the doctor to make the centripetal movement towards the nurse's perspective, she engages in an opposite movement towards the perspective that the doctor entertained in Phase I. This double movement leaves the doctor in a tense position: if she picks up the embodied information and is convinced by the nurse's scenario, she has to ignore the nurse's subsequent estimation - and vice versa. Placed in this tension, the doctor hesitates (cf. the pause of 0.7 seconds in line 14) as she makes a noticeably facial expression that resembles a kind of lip-pursing grimace response (see Figure 5). The doctor does not respond verbally, but she wrinkles her nose and pouts her lips in a remarkable way.

Figure 5. The doctor's lip-pursing grimace in line 14. For purposes of clarity, we have removed minor jpeg compression artefacts around the lips of the subject

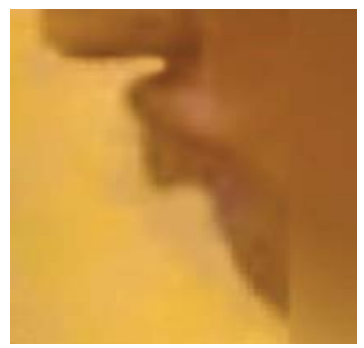


While we cannot determine the meaning embedded in this grimacing, we can extrapolate it from her subsequent behaviour, in particular the fact that later in the sequence she attempts to align with the nurse's position, arguing that the nurse is the most experienced of the two (line 32). If the doctor's meaning trajectory changes through the seven phases, this particular moment is a viable candidate for the first occurrence of some sort of concern pertaining to her initial position. If so, the embodied knowledge of the nurse turns out to be an important affordance for the doctor to change her mind. The nurse's expertise shows in the way she manipulates the affordances in the environment of the dialogical system: She indicates what is relevant through embodied behaviour (gaze, movements, and wordings), and the meaning that emerges is a direct result of this embodied expertise. Encountering the nurse's affordances for meaningmaking, the doctor freezes, as she is prompted to reconsider the consequences of her initial decision. Since the doctor does not respond directly to her utterance in line 13, the nurse continues her turn by remove by explicating that that in line 13 refers to plac[ing] a bedpan under when you need to go to the toilet and such (line 15). As she now directs her turn to the patient, she shifts from the doctor-nurse we (line 13), to the patient you (line 15).

The patient also picks up on the nurse's visualisation. While she does not have the medical expertise to decide whether it is possible to place the bedpan or not, the nurse's bodily visualisation has a strong illustrative power that enables her to imagine the situation in a tangible way beyond verbal explanations. Obviously, to wiggle one's hip and torso is not a preferable activity with a hip fracture, and the nurse's whole-bodied meaning-making prompts the patient to utter her doubt about whether this is doable or not (line 16). However, for the second time the nurse counters her own objections. This time she compares general cases with the patient in question: but it seems like you are pretty good anyway to just get (line 16).

As we reach the end of Phase II, we see that both the doctor and the nurse have moved from their initial position to a position characterised by doubt and meaning equivocality. Both agents have formulated immediate interpretations of the situation, and both agents have undergone changes in their perspective, which results in confusion, because in the end only one decision can be made. Ideally, a necessary step is to align and calibrate the system so that it is able to act based on a shared meaning. In this case, however, the attempt to do so has created even more confusion and insecurity in the dialogical system.

\section{Phase III: Emergent changes in perspectives}

The dialogue so far has led to a point where the nurse doubts her own suggestion, the doctor doubts her rejection of the nurse's suggestion, and the patient expresses uncertainty and insecurity. In Phase III, which covers a small excerpt in the transcript (line 19-23), the doctor voices her last attempt to legitimise her 
rejection of the nurse's suggestion. She is prompted to do so by a short exchange between the doctor and the patient: the patient informs the medical team that her hip hurts (this one it hurts, line 19), and the doctor responds that the FIC block will remove the pain. Her conclusion from this exchange is expressed as a hesitant request for confirmation: don't you don't you think we manage ${ }^{\circ o}$ without ${ }^{\circ o}$ (line 23). That they can manage the situation without catheterisation is thus linked to the FIC block, but that is another fallacious conclusion: just as the fact that the patient does not normally use catheter does not warrant the conclusion that she should not be catheterised, so the fact that the patient will be anaesthetised does not necessarily warrant the conclusion that catheterisation is not needed. Given that the doctor less than three seconds later in fact expresses a concern with her own pre-treatment administration, her hesitancy may indicate that she is in a process of realising that her argument is flawed: the relevant clinical question is whether the patient will be sufficiently medicated to permit the bedpan placement without hurting the patient.

An experienced emergency medical practitioner, such as the nurse, knows that already. But insisting on bringing these considerations to the fore in the medical team's meaning-making would conflict with the doctor's reliance on patient-provided information, and it would also disturb the delicate balance between being the experienced practitioner and being the inferior in the role hierarchy. As a consequence, the nurse has been silenced, and she abstains from explicating her opinion any further. Instead, she replies with two minimally responsive yes (line 20 and 22) to both the patient's and the doctor's utterances. This dialogical behaviour where her meaning is withdrawn from the systemic reasoning altogether changes the interactional dynamics further. Thus, while the doctor's request for confirmation in line 23 functions as a conclusion, it also calls for the nurse to take a more active role in the meaning-making process.

At this point in the cognitive trajectory, the coordination in the dialogical system is constrained and this constraint inhibits the system in moving on. In order to get back on track, the system needs to recalibrate and dampen its meaning oscillations: they must agree on what to do next. Such a decision requires either powerful autonomy, where a single agent overrules the other and simply decides what to do, or a shared meaning-making process where the dialogical system as a whole decides what to do.

\section{Phase IV: Cultural dynamics in situated meaning-making}

In Phase IV (line 24), these two states of individual autonomy and system autonomy co-exist as a tension in the dialogical system. The phase consists of only one utterance, and it is defined as a distinct phase because the line of argumentation changes: for the first time in the exchange, the general procedures in the department become part of the meaning-making. This happens as the nurse refers to catheterisation as a standard procedure at the department: 
we often do it (line 24). She draws on non-local resources (Steffensen, 2015) in the dialogical system's meaning-making, as the pronoun we evokes a general team of professionals (Linell, 2009) who often choose to catheterise. This strategy scaffolds her situated meaning-making by drawing on experience and general practice. Hollan et al. (2000:178) elaborate:

Culture is a process that accumulates partial solutions to frequently encountered problems. Without this residue of previous activity, we would all have to find solutions from scratch. We could not build on the success of others. [...] This is tremendously enabling. But it is not without cost. For culture may also blind us to other ways of thinking, leading us to believe that certain things are impossible when in fact they are possible when viewed differently.

The power of culture is re-enacted, as the nurse appropriates sociocultural voices, and as such she introduces a powerful perspective on the dialogical system's joint meaning-making. After all, the widespread use of catheterisation is an important perspective for a novice doctor who has no experience with this part of the pre-treatment. As such, the nurse makes non-local aspects of general practices highly relevant.

Per se, the nurse seems to attempt to dissolve the dialogical system's impasse by empowering the system by providing useful information. But the nurse's reply is ambiguous: immediately after this prioritisation of systemic autonomy, she undermines the system's joint meaning-making by downplaying the importance of the department's general procedures. She does so by scaffolding the novice doctor's individual autonomy in an explicit support for the doctor's plan: but let us just try and see (line 24). The ambiguity is striking: on the one hand, the nurse speaks with an authority grounded in her knowledge and expertise, which calls for catheterisation; and on the other hand, she argues against this decision.

\section{Phase V: The doctor and the nurse swop positions}

How does a sensitive dialogical partner react to such ambiguity? The doctor has the nurse's support to a pre-treatment plan that does not include catheterisation, but at the same time the doctor is aware that this plan goes against departmental procedures. The result is striking: in Phase V (line 25-27) the doctor and the nurse swop positions! The nurse complies with the non-catheterisation plan, arguing from the relative mobility of the patient, and the doctor shelves her initial conviction, explicitly picking up on the nurse's reference to departmental procedures: it it is well it might be that we should that is you eh (line 25). So at the very point in time where the doctor accepts the nurse's suggestion, the nurse withdraws her suggestion on the pretext of identifying the patient as relatively mobile, even though the 92-year-old patient is in a great deal of pain and complains about her physical condition several times (line 19 and 28). As a result, both the doctor and 
the nurse have made a meaning U-turn based on their information pick-up and on the oscillations in the dialogical system.

The nurse has drawn the doctor's attention to social affordances in the environment, and the doctor has enacted her lack of expertise. While this change could have led to a systemic reconfiguration where they decide on a new and better pre-treatment plan, the outcome is different: thus, in the next two phases, the doctor's confession of her inexperience becomes an affordance for the nurse to maintain the doctor's systemic position - and her face (Goffman, 1959).

\section{Phase VI-VII: Functionality and dysfunctionality in the dialogical system}

During the overall event, multiple suggestions have been evaluated within the team, and both their meaning-making and their positioning in the dialogical system have undergone significant changes. The result of these oscillations in the dialogical system is the emergence of a decision: In Phase VI (line 31-32) the nurse concludes: let us just eh (.) now we are ju:st trying to give this so (line 31) and shortly after the doctor explicitly refers to the nurse as the expert: $o$ - or thisI think it is you who a:re the mo::st eh (line 32). In Phase VII (line 33) the meaning-making trajectory reads a temporary end point in the shape of a publicly stated decision: we will just wait and see (0.3) because I actually think that eh it is realistic and. This decision is the cognitive result of 42.2 seconds of reasoning. It functions as the primary event pivot in the sequence, as this decision defines the overall sequence as one of meaning-making and reasoning.

From these two phases, the self-organising nature of the decision-making process is evident: The decision is not the result of any pre-conceived pre-treatment plans that the practitioners try to realise. Indeed, both agents in the system abandon their initial arguments and come to sponsor the intuitions of the other agent (cf. Figure 6).

Figure 6. The figure illustrates how the two agents move through a logical space between the decision terms: catheterisation and non-catheterisation. The figure is not intended as a quasi-mathematical model, but rather as a heuristic illustration of movements between the two decision terms

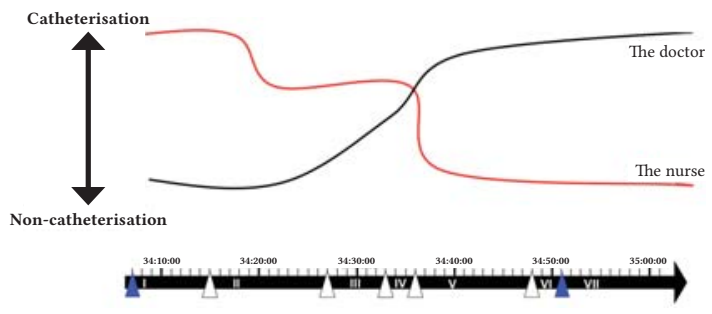


This oscillation indicates that the decision is less the result of medically informed reasoning, and more a result of how the role hierarchy and the protection of professional face take the lead. Seen from a strictly functional point of view - as favoured in the tradition of Distributed Cognition (Hutchins, 1995; Perry, 2010) - the system becomes dysfunctional because it allows task irrelevant considerations to constrain the reasoning process. The two practitioners mesh their inter-bodily dynamics with experience and medical expertise, and this process gives rise to meaning and decisions, as well as to insecurity, confusion, and frustration. The dialogical system thus has to balance medical decision-making and interpersonal emotional processes. As can be seen from Figure 6, these dynamics lead to a decision that is predominantly determined by the position that the nurse takes at the end of the sequence. In the dynamics of the dialogical system, the nurse takes the lead and secures that a decision is made. But the price is high, for at the same time she puts herself in a non-expert position by withdrawing her experience-based proposal and deciding to do what the doctor initially argued.

From a purely medical point of view the decision is not based on best practice and expertise but on interpersonal caring and an orientation to hierarchies and practices for decision-making. The case demonstrates that in real-life situations, decisions are not made in a vacuum but embedded in meaningful interpersonal relations shaped through processes of maintaining each other's face (Goffman, 1959) and orienting to role hierarchies that exist between different professional groups (Pedersen, 2010).

\section{Epilogue: on the poverty of phenomenal reports}

A few hours after the recordings, both the doctor and the nurse were interviewed as part of the ethnographic project at the ward. During her interview, the doctor told the researcher that "the nurse was right." It turned out that it was not possible to place the bedpan under the patient, and they had to insert the catheter at a later and more complicated stage in the pre-treatment process. This caused a delay in the treatment plan, and it harmed the patient unnecessarily. According to the doctor, she was wrong and the nurse was right: "as the nurse also said," she explained, catheterisation did indeed turn out to be necessary.

Evidently, the doctor did not experience the situation as shared decision-making with equally shared responsibility for the decision that they reached: she identified herself as the main responsible for the decision. Such an approach to decision-making is widespread in the medical world: "Errors are regarded as someone's fault, caused by a lack of sufficient attention or, worse, lack of caring enough to make sure you are correct. [...]. While the proximal error leading to an accident is, in fact, usually a 'human error,' the causes of that error are often well beyond the individual's control” (Leape, 1994:1852). 
The nurse reached a similar conclusion in her post-treatment interview: the collaboration went well, but as it is often the case, the doctor had not listened carefully to what she, as a nurse, had to say. Thus, in the post hoc interviews, both the doctor and the nurse reach the same conclusion about responsibility: the nurse was right about the work procedure, but she was overruled by the doctor. However, their experience of their team performance does not match the actual coordination shown in the data. Initially, the doctor rejected the nurse's proposal, but that changed as the nurse informed the doctor about possible future complications and departmental procedures. Thus, from the data we identified a gradual change of perspective in the doctor's meaning-making, even to the degree that she realises that the nurse, as the more experienced of the two, should take the lead: I think it is you who a::re the mo::st eh (line 32). The fact that they both report a sequence of events that does not match their behaviour in situ is a powerful testimony to the fragility of phenomenal reports: the widespread cultural narrative of dominant doctors and inferior nurses frame their interpretation of the events they went through a few hours earlier.

\section{Conclusion}

What is so striking about the catheterisation case is, first, that it exemplifies decision-making without a singular agent making the decision, and, second, that it points to the importance of non-local processes in local decision-making. As for the first of these points, if rationality was unbounded (cf. Secchi, 2011), a decision-making process could in principle be completely computational and logical. But since that is not the case, decision-making relies on meaning-making processes. The scrutiny of the example has shown that even though the two main cognisers are two meaning-making individuals, it is the dynamics within the overall dialogical system that leads to the decision not to catheterise. Thus, the cognitive outcome of the process is a result of how the two practitioners oscillate between being a multi-agent-system with shared, tightly coordinated agency and a loosely coupled dialogical system where the individual brings forth an understanding based on each their professional background and each their expertise. Joint meaning-making thus emerges as the agents shift between centripetal and centrifugal dynamics in the system, e.g. by (a) pointing to relevant affordances in the environment, so new meaning emerges as new information is picked up and made relevant for the whole system; (b) by enacting and embodying their medical-cultural knowledge in the situation, which allows for evidence-based meaning-making and decision-making in the dialogical system, and (c) by evaluating the meaning that emerges in the dialogical system.

Secondly, taking an ecological perspective on the meaning-making processes, the example reveals how cultural dynamics affect local decision-making. Culture transcends individual reasoning, and it is constantly re-enacted as an emergent 
phenomenon. Decision-making processes are shaped by previous episodes, so that the practitioners' actions reflect embodied understandings of multiple cultural phenomena, for instance role hierarchies, systemic norms, physical and procedural limitations, personal experience, and caring for human beings in complex situations.

In conclusion, when one studies cognitive dynamics in dialogical systems, one has to take into consideration how the system is saturated by (a) the agents' non-local experiences of sociocultural and institutional procedures, (b) the team's compliance with non-local expectations about how decision-making is managed (role hierarchies etc.), and (c) situated affordances for specific actions. Crucial to a team's general successfulness is the ability to balance procedures and interpersonal dynamics by caring for both the persons in the dialogical system (in our case, patients, colleagues and oneself) and the task to be solved by the system (in our case, diagnosis, examination, and treatment).

In this article, we have analysed an emergent decision as interwoven with meaning-making processes in a dialogical system. In so doing, we have suggested that an ecological approach traces interactional meaning to the dynamics within the dialogical system, in particular how the agents recalibrate the system by shifting between a systemic mode and an individual mode.

From a traditional linguistic point of view, this approach to meaning is (perhaps unacceptably) radical, as it implies that human beings can engage in long stretches of interaction without any significant meaning-making, as long as the dialogical system is sufficiently stable (and hence not being recalibrated). But one has to distinguish between, on the one hand, what an observer can describe as meaning(ful), and, on the other, what 'meaning' is from an ecological point of view. Thus, it is hardly surprising that we can describe entities as bearing meaning; after all, in literate societies we all know the practice of consulting a dictionary. ${ }^{6}$ On this view, it is tempting to assume that each symbol that can be listed in the dictionary thus has a unique meaning (or meaning potential) that can be used (or instantiated) in human interaction; we may even assume that we all carry with us an inner lexicon that contains more or less the same information as the dictionary. But at the end of the day, this point of view depends on the assumption that human cognition is an inner machinery that gives us "competence" or "knowledge," which in turn give rise to "performance" and "use" - and while space prevents us from rehearsing the arguments against it, it is simply an unacceptable assumption from the point of view of ecological cognitive science. But indeed one can ask - as one of our reviewers did - what is the role of meaning in communication if 'meaning' is exclusively traced to recalibrations in dialogical-cognitive systems? The fallacy underlying such a question is that we readily assume that meaning is necessarily at play whenever we engage in the

\footnotetext{
${ }^{6}$ For instance, we can read in the Oxford English Dictionary that "meaning" means "what is meant by a word, text, concept, or action."
} 
phenomenon called 'language'. But considering the wide array of automatised, routinely performed behaviours we are capable of - peeling potatoes, mowing the lawn, humming Roger Whittaker tunes - why should everyday talk be any different? Why do we want even the most mundane verbal activities - say the 30 minutes of chit-chat at the hairdresser - to be the result of a unique human capacity for meaning-making?

From an ecological point of view, the hairdresser example does indeed rely on meaning: As an exercise of presenting our everyday self (Goffman, 1959), we coordinate our behaviour with the concrete task at hand, and this coordination depends on such meaning-making processes as agreeing on a desired output, sitting still, and engaging in small-talk, which all depend on an initial recalibration of the dialogical system. However, once that has been achieved, the actual utterances throughout the hairdressing procedure do not require much meaning-making. Not unlike how bird vocalisation contributes to complex social behaviour without symbolic meaning, human vocalisation can contribute to social grooming in ways where "the content" of what is actually said does not matter much. Meaning, on this view, is an interpretational attitude - or an enacted interpretation - that allows us to modify organism-environment relations in ways that do not require much effort. As such, meaning is a mechanism that allows us to mesh real-time biological agency with non-local resources (Steffensen, 2015). Meaning is thus neither the content of semiotic symbols, nor a feature of individual cognitive processes. Rather, meaning is induced when cognitive agents recalibrate dialogical-cognitive systems, and this process can be traced to the interplay between fast, enchronic timescale of the interaction (Enfield, 2014) and slow sociocultural timescales of institutions and social systems.

These theoretical considerations also underlie the methodological approach pursued in this article. Methods for studying social interaction normally prioritise what happens in real-time, and this fixation on one local timescale (Pedersen, 2015; Steffensen and Pedersen, 2014) often leads to a simplistic understanding, because it reduces the complexity of human behaviour to a few causal mechanisms. If indeed meaning is animated by human beings in a sense-saturated ecology (Steffensen, 2011), we need methods that allow us to trace meaning-making processes to ecosystemic activity, and methods that allow us to investigate behavioural outcomes as shaped by both non-local dynamics and situational affordances for actions within the dialogical system. In this article, we have presented the method of Cognitive Event Analysis as such an ecological method.

CEA focuses on how disturbances, fixations and other constraints on action affect functional coordination. CEA-style investigations of the dynamical coordination go beyond the enchronic scale of interaction, and it thus allows for an integration with theoretical perspectives on human cognitive, emotional, and linguistic capabilities for action, as well as with the moral obligations that prompt human beings to engage in certain behaviours rather than in others. 
However, CEA does not equip us with a comprehensive basis for answering, say, why a patient interruption prompts practitioners to either ignore or respond to the comment. CEA cannot explain what constitutes enabling conditions, but it can point to them as conditions for meaning-making that leads to results. Arguably, a method for investigating how human beings animate dialogical systems in order to achieve results is not only valuable for interaction analysts and cognitive psychologists. Sociologists, anthropologists, and other social scientists may also benefit from methods that allow them to study the texture of the interface between human agents and their environment.

\section{References}

Bühler, K. (1934). Sprachtheorie: Die Darstellungsfunktion der Sprache. Jena: Verlag von Gustav Fischer.

Cowley, S.J. (Ed.) (2011). Distributed Language. Amsterdam: John Benjamins.

Cowley, S.J. \& Nash, L. (2013). Language, interactivity and solution probing: Repetition without repetition. Adaptive Behavior, 21 (3), 187-198.

Cuffari, E., Di Paolo, E., \& De Jaegher, H. (2015). From participatory sense-making to language: there and back again. Phenomenology and the Cognitive Sciences, 14 (4), 1089-1125.

De Jaegher, H. \& Di Paolo, E. (2007). Participatory sense-making. Phenomenology and the Cognitive Sciences, 6 (4), 485-507.

Enfield, N.P. (2014). Causal Frames for Understanding Language. In N.J. Enfield, P. Kockelman, \& J. Sidnell (Eds.), Cambridge Handbook for Linguistic Anthropology. Cambridge: Cambridge University Press.

Fowler, C.A., Richardson, M.J., Marsh, K.L., \& Shockley, K.D. (2008). Language use, coordination, and the emergence of cooperative action. In A. Fuchs \& V.K. Jirsa (Eds.), Coordination: Neural, Behavioral and Social Dynamics (pp. 261-279). Dordrecht: Springer.

Fusaroli, R. \& Tylén, K. (2012). Carving language for social coordination: A dynamical approach. Interaction Studies, 13 (1), 103-124.

Gibson, J.J. (1979). The Ecological Approach to Visual Perception. Boston: Houghton Mifflin.

Goffman, E. (1959). The Presentation of Self in Everyday Life. London: Penguin Books.

Harvey, M.I., Gahrn-Andersen, R., \& Steffensen, S.V. (2016). Interactivity and enaction in human cognition. Constructivist Foundations, 11 (2), 602-613.

Hollan, J., Hutchins, E., \& Kirsh, D. (2000). Distributed cognition: toward a new foundation for human-computer interaction research. ACM Transactions on Computer-Human Interaction (TOCHI), 7 (2), 174-196.

Hutchins, E. (1995). How a Cockpit Remembers Its Speeds. Cognitive Science, 19(3), 265-288. 
Jefferson, G. (2004). Glossary of transcript symbols with an introduction. In G.H. Lerner (Ed.), Conversation Analysis: Studies from the First Generation (pp. 13-31). Amsterdam: John Benjamins Publishing Company.

Järvilehto, T. (1998). The theory of the organism-environment system: I. Description of the theory. Integrative Physiological and Behavioral Science, 33 (4), 321-334.

Järvilehto, T. (2009). The Theory of the Organism-Environment System as a Basis of Experimental Work in Psychology. Ecological Psychology, 21 (2), 112-120.

Konvalinka, I., Xygalatas, D., Bulbulia, J., Schjodt, U., Jegindo, E.M., Wallot, S., Orden, G.V., Roepstorff, A. (2011). Synchronized arousal between performers and related spectators in a fire-walking ritual. Proc Natl Acad Sci U S A, 108 (20), 8514-8519.

Kravchenko, A.V. (2007). Essential properties of language, or, why language is not a code. Language Sciences, 29 (5), 650-671.

Lave, J. \& Wenger, E. (1991). Situated Learning: Legitimate Peripheral Participation. Cambridge: Cambridge University Press.

Linell, P. (2009). Rethinking Language, Mind, and World Dialogically: Interactional and Contextual Theories of Human Sense-Making. Charlotte, NC: Information AgePublishing. Linell, P. (2013). Distributed language theory, with or without dialogue. Language Sciences, 40, 168-173.

Linell, P. (2015). Dialogism and the Distributed Language Approach: A Rejoinder to Steffensen. Language Sciences, 50, 120-126.

Love, N. (2007). Are languages digital codes? Language Sciences, 29 (5), 690-709.

Marsh, K.L., Johnston, L., Richardson, M.J., \& Schmidt, R. (2009). Toward a radically embodied, embedded social psychology. European fournal of Social Psychology, 39 (7), 1217-1225.

Marsh, K.L., Richardson, M.J., \& Schmidt, R.C. (2009). Social connection through joint action and interpersonal coordination. Topics in Cognitive Science, 1(2), 320-339.

Pedersen, S.B. (2012). Interactivity in health care: bodies, values and dynamics. Language Sciences, 34 (5), 532-542.

Pedersen, S.B. (2015). The Cognitive Ecology of Human Errors in Emergency Medicine: An Interactivity-Based Approach. (Ph.D.-afhandling). Odense: University of Southern Denmark.

Pedersen, S.B. \& Steffensen, S.V. (2014). Temporal Dynamics in Medical Visual Systems. Cybernetics \& Human Knowing, 21 (1-2), 143-157.

Perry, M. (2010). Socially distributed cognition in loosely coupled systems. Ai \& Society, 25 (4), 387-400.

Rączaszek-Leonardi, J. (2011). Symbols as constraints: The structuring role of dynamics and self-organization in natural language. In S.J. Cowley (Ed.), Distributed Language (pp. 161-184). Amsterdam: John Benjamins.

Rączaszek-Leonardi, J. \& Cowley, S.J. (2012). The evolution of language as controlled collectivity. Interaction Studies, 13 (1), 1-16. 
Rączaszek-Leonardi, J., Dębska, A., \& Sochanowicz, A. (2014). Pooling theground: understanding and coordination in collective sense making. Frontiers in Psychology, 5 (1233), 1-13.

Richardson, M.J., Marsh, K.L., \& Baron, R.M. (2007). Judging and actualizing intrapersonal and interpersonal affordances. Fournal of Experimental Psychology: Human Perception and Performance, 33 (4), 845-859.

Secchi, D. (2011). Extendable Rationality: Understanding Decision Making in Organizations. New York: Springer.

Sidnell, J. (2016). Interactional trouble and the ecology of meaning. Psychology of Language and Communication, 20 (2), 98-111.

Steffensen, S.V. (2012). Care and conversing in dialogical systems. Language Sciences, 34 (5), 513-531.

Steffensen, S.V. (2013). Human interactivity: Problem-solving, solution-probing and verbal patterns in the wild. In S.J. Cowley \& F. Vallée-Tourangeau (Eds.), Cognition Beyond the Brain: Computation, Interactivity and Human Artifice (pp. 195-221). Dordrecht: Springer.

Steffensen, S.V. (2015). Distributed Language and Dialogism: notes on non-locality, sense-making and interactivity. Language Sciences, 50, 105-119.

Steffensen, S.V. (2016). Cognitive probatonics: Towards an ecological psychology of cognitive particulars. New Ideas in Psychology, 42, 29-38.

Steffensen, S.V. \& Pedersen, S.B. (2014). Temporal Dynamics in Human Interaction. Cybernetics \& Human Knowing, 21 (1-2), 80-97.

Steffensen, S.V., Vallée-Tourangeau, F., \& Vallée-Tourangeau, G. (2016). Cognitive events in a problem-solving task: a qualitative method for investigating interactivity in the 17 Animals problem. Fournal of Cognitive Psychology, 28 (1), 79-105.

Trasmundi, S.B. \& Linell, P. (submitted). Interactivity, extended dialogism and cognitive event analysis: finding problems to solutions in emergency medicine.

Tylén, K., Weed, E., Wallentin, M., Roepstorff, A., \& Frith, C.D. (2010). Language as a tool for interacting minds. Mind \& Language, 25 (1), 3-29.

Vallée-Tourangeau, F., Steffensen, S.V., Vallée-Tourangeau, G., \& Makri, A. (2015). Insight and Cognitive Ecosystems. Paper Presented at the Proceedings of the Thirty-seventh Annual Conference of the Cognitive Science Society. Austin, TX: Cognitive Science Society.

Van Orden, G.C., Holden, J.G., \& Turvey, M.T. (2003). Self-organization of cognitive performance. Fournal of Experimental Psychology: General, 132 (3), 331-350.

Van Orden, G.C., Kloos, H., \& Wallot, S. (2011). Living in the pink: Intentionality, wellbeing, and complexity. In C. Hooker (Ed.), Philosophy of Complex Systems. Amsterdam: Elsevier.

Wilson, R.A. \& Clark, A. (2009). How to situate cognition: Letting nature take its course. In M. Aydede \& P. Robbins (Eds.), The Cambridge Handbook of Situated Cognition. Cambridge: Cambridge University Press. 